\title{
Sex-related psychological effects on metabolic control in type 2 diabetes mellitus
}

\author{
G. Kacerovsky-Bielesz • S. Lienhardt • M. Hagenhofer • \\ M. Kacerovsky • E. Forster • R. Roth • M. Roden
}

Received: 18 November 2008 / Accepted: 8 February 2009/Published online: 10 March 2009

(C) Springer-Verlag 2009

\begin{abstract}
Aims/hypothesis Women are at higher risk of diabetesrelated cardiovascular complications than men. We tested the hypothesis that there are sex-specific differences in glucometabolic control, and in social and psychological factors. We also examined the influence of these factors on glucometabolic control.

Methods We examined 257 (126 men/131 women) consecutive patients ( $64 \pm 9$ years, means \pm SD) of a metropolitan diabetes outpatient service employing clinical testing and standardised psychological questionnaires.

Results Mean $\mathrm{HbA}_{1 \mathrm{c}}(7.6 \pm 1.2 \%)$ was not different between women and men. Women patients on oral hypoglycaemic agents were better informed about diabetes $(p=0.012)$. They employed more strategies for coping with diabetes, including
\end{abstract}

G. Kacerovsky-Bielesz $\cdot$ M. Roden

Medical Department, Hanusch Hospital,

Vienna, Austria

G. Kacerovsky-Bielesz $\cdot$ M. Kacerovsky $\cdot$ M. Roden

Karl-Landsteiner Institute for Endocrinology and Metabolism,

Vienna, Austria

S. Lienhardt $\cdot$ M. Hagenhofer $\cdot$ R. Roth

Institute for Psychology, Karl Franzens University,

Graz, Austria

E. Forster

Department of Laboratory Medicine, Hanusch Hospital,

Vienna, Austria

M. Roden $(\bowtie)$

Institute for Clinical Diabetology,

German Diabetes Center (Leibniz Center for Diabetes Research)

at Heinrich Heine University,

Auf'm Hennekamp 65,

40225 Düsseldorf, Germany

e-mail: michael.roden@ddz.uni-duesseldorf.de religion $(p=0.0001)$, active coping $(p=0.048)$ and distraction $(p=0.007)$. Women reported lower satisfaction with social support $(p=0.034)$, but not more depression than men. Although no differences were observed in compliance, insulin-treated patients were more satisfied with their therapy $(p=0.007)$. Variables predicting poor metabolic control were different in men $\left(R^{2}=0.737, p=0.012\right)$ and women $\left(R^{2}=0.597\right.$, $p=0.019$ ). Major predictors of high $\mathrm{HbA}_{1 \mathrm{c}}$ included depressive coping, lower sexual desire, quality of life and internal locus of control, but high external doctor-related locus of control in women and frequent emotional experiences of hyperglycaemia in men.

Conclusions/interpretation Lower quality of life, internal control and socioeconomic status, and higher prevalence of negative emotions probably prevented woman patients from achieving improved glucose control despite their better knowledge of and greater efforts to cope with diabetes. We suggest that women patients would benefit from individualised diabetes care offering social support, whereas men would benefit from knowledge-based diabetes management giving them more informational and instrumental support.

Keywords Compliance · Depression · Gender - Glucose . Quality of life $\cdot$ Sex $\cdot$ Type 2 diabetes mellitus

$\begin{array}{ll}\text { Abbreviations } \\ \text { BSSS } & \text { Berlin Social Support Scales } \\ \text { DBP } & \text { Diastolic BP } \\ \text { DTSQ } & \text { Diabetes Treatment Satisfaction Questionnaire } \\ \text { FKV } & \text { Freiburger Illness Coping Scale } \\ \text { FPG } & \text { Fasting plasma glucose } \\ \text { FPI } & \text { Fasting plasma insulin } \\ \text { GLA } & \text { Glucose-lowering agents } \\ \text { ICP-D1 } & \text { Diabetes Locus of Control Questionnaire } \\ \text { MANOVA } & \text { Multivariate three-factorial ANOVA }\end{array}$




$\begin{array}{ll}\text { MAS } & \text { Marital Adjustment Scales } \\ \text { NHP } & \text { Nottingham Health Profile } \\ \text { GLAPLD } & \text { Problem Areas in Life with Diabetes } \\ \text { QUICKI } & \text { Insulin sensitivity check index } \\ \text { SBP } & \text { Systolic BP }\end{array}$

\section{Introduction}

Women are at increased risk of diabetes-related cardiovascular complications [1]. Behavioural patterns including less favourable lifestyle account for $\sim 40 \%$ of premature deaths [2]. Again, diabetic women are at a more than 1.5 -fold and threefold higher relative risk of a fatal outcome of coronary heart disease than diabetic men and non-diabetic women, respectively [3].

Despite these striking sex-related differences, the underlying sex-specific aspects of behavioural pattern have not been sufficiently addressed. Studies in diabetic patients have shown that increased systolic BP (SBP), smoking and alcohol consumption seem to be more frequent in men, whereas hyperuricaemia and low habitual physical activity are more common in women [4]. There might also be sex-specific differences in inflammatory and obesity-related markers determining type 2 diabetes risk [4]. Recently, evidence was provided that diabetic women with cardiovascular disease have poorer control of modifiable risk factors and receive less intensified lipid-lowering treatment than men [5].

Psychological factors have an important impact on therapy adherence and control in chronic diseases. Interestingly, the prevalence of anxiety is almost doubled in type 2 diabetic patients compared with healthy individuals [6]. Depression is twice as common in diabetic patients than in non-diabetic individuals [7]. Elderly, obese women with diabetes-related complications might have lower overall quality of life [7], which is likely to be related to lower education, income and physical activity in US study cohorts [8]. Detailed information on how sex-specific psychosocial variables impact on metabolic control is not yet available. Although patient-oriented education geared to selfmanagement improves treatment adherence and glycaemic control in patients with type 2 diabetes [9], it is still unclear whether sex-specific differences affect compliance with diabetes management and/or strategies for coping with the disease, including depressive or active approach, problemoriented behaviour or distraction, and religiosity or wishful thinking [10].

Thus, the present study tested the hypothesis that sexspecific differences exist in terms of (1) glucometabolic control and (2) social and psychological factors. We also assessed how these factors influence glucometabolic control as measured by $\mathrm{HbA}_{1 \mathrm{c}}$ in patients of a metropolitan diabetes outpatients' centre.

\section{Methods}

Patients and protocol We enrolled 257 consecutive patients who had been seen in the diabetes outpatient clinic at least twice during the preceding 6 months. Further inclusion criteria were 40 to 80 years of age, known type 2 diabetes and the ability to read and fill in all questionnaires. All of the 265 outpatients who fulfilled these criteria and were seen in the centre during a period of 3 months were approached. Only eight patients refused participation because of lack of time or interest in the study. The basic demographics of those who declined were not different from those included in the study. All participants gave written informed consent to the study protocol, which was approved by the institutional ethics boards and performed in accordance with the most recent version of the Declaration of Helsinki.

In addition to the patients' histories, detailed sociodemographic information on marital status, living and job situation, and on the highest level of education was recorded. Diabetes-specific information included known diabetes duration, nutritional habits, frequency of blood glucose self-control, hyperglycaemic/hypoglycaemic events, number of tablets of oral glucose-lowering agents (GLA) or insulin injections per day, attendance of diabetes education courses and hospitalisation. The clinical examination comprised measurements of waist circumference, body height and weight for computation of BMI, and systolic and diastolic BP (DBP) measured at rest in the upright sitting position. Blood was drawn from all participants after $12 \mathrm{~h}$ of fasting to measure fasting plasma glucose (FPG), fasting plasma insulin (FPI), C-peptide, $\mathrm{HbA}_{1 \mathrm{c}}$, triacylglycerol and total, HDL- and LDL-cholesterol. Thereafter, patients completed a series of psychological questionnaires, which were explained in detail to them by two psychologists who were also available for further explanation, debriefing and monitoring any signs of fatigue. The participants had several hours to complete this task and were given breaks between tests. Anonymity was assured for all data collected and stored.

Laboratory tests and calculations Glucose was measured with the hexokinase method (Abbott Aeroset, Abbott Park, IL, USA), insulin and C-peptide with an immunoluminescence method (DPC/Siemens/Immulite, Flanders, NJ, USA) and $\mathrm{HbA}_{1 \mathrm{c}}$ by HPLC (Biorad, Hercules, FL, USA). Triacylglycerol, total cholesterol, and HDL- and LDL-cholesterol were assayed using automated routine laboratory methods (LN09D05-01, A1522460; Abbot Aeroset). Fasting insulin sensitivity was assessed from FPI and FPG as the insulin sensitivity check index (QUICKI) using the term $1 /(\log$ $[\mathrm{FPG}]+\log [\mathrm{FPI}])$ [11]. The triacylglycerol:HDL-cholesterol ratio served as a surrogate index of insulin resistance [11]. 
Psychological questionnaires Standardised questionnaires were presented to all participants. They were partly developed and validated for diabetic populations and/or are frequently used in diabetic cohorts. All questionnaires were in German and addressed the areas described below.

The tests on knowledge of diabetes and treatment differed between diabetic patients, according to whether they were on GLA [12] or on insulin treatment [13]. The knowledge test for GLA in type 2 patients [12] contains 14 items; the correct answers are counted and the total score varies between 0 and 14. The knowledge test for patients on insulin [13] contains 40 items; here, too, the correct answers are counted, with the total score varying between 0 and 40 .

Coping with the disease was measured by the Freiburger Illness Coping Scales (FKV) [14]. This questionnaire consists of 35 items on five factors that represent commonly used strategies for coping with disease: (1) depressive coping style, (2) active, problem-oriented coping, (3) distraction, (4) religiosity and (5) trivialising and wishful thinking. The items are endorsed on a five-point scale from 'not at all-1' to 'always - 5', with a score of 25 reachable for each factor.

The Problem Areas in Life with Diabetes (PLD) questionnaire [15] is composed of 18 items, which can be answered on a five-point scale from 'not at all-1' to 'very strong-5'.

Control beliefs related to diabetes were measured by the Diabetes Locus of Control Questionnaire (ICP-D1) [16]. This questionnaire assesses which sources account for the consequences of a specific behaviour and contains 29 items, endorsed on a seven-point scale from 'not at all -3 ', to 'exactly +3 ', related to four factors: (1) internality, (2) physician-related externality, (3) unpredictability and (4) chance.

Quality of life was assessed by the German version of the Nottingham Health Profile (NHP) [17], consisting of 38 items (rated yes or no) that constitute six scales: (1) loss of energy, (2) pain, (3) emotional reactions, (4) sleep, (5) social isolation and (6) physical mobility.

Social support was measured by the Berlin Social Support Scales (BSSS) [10] with 15 items, rated on a fourpoint scale from '1, don't agree' to '4, agree completely', constituting four factors: (1) emotional support, (2) instrumental support, (3) informational support and (4) satisfaction with support.

The Marital Adjustment Scales (MAS) [18] were presented to measure the quality of partnership by a total of nine items. The first item considered happiness ('very happy, 1' to 'very unhappy, 7', where 4 means as happy as most people). The remaining eight items assessed aspects of partnership such as decisions on money, leisure time etc. on a six-point scale from 'we agree on this issue completely 1' to 'we disagree completely 6'.
Satisfaction with therapy was assessed with the Diabetes Treatment Satisfaction Questionnaire (DTSQ) [19]. Six items cover therapy satisfaction and two separate items deal with experience of hypoglycaemia and hyperglycaemia respectively. The items are endorsed on a seven-point scale from 0 ('never') to 6 ('always') and 0 ('dissatisfied') to 6 ('satisfied').

Compliance with treatment was assessed by a single item 'I follow the therapy recommendations' on a five-point rating, ranging from 1 ('absolutely accurate') to 5 ('not at all'). Erectile function was evaluated with a two-item scale testing whether an erection can be achieved and maintained, and the frequency of erections on a five-point rating scale ranging from 1 ('not at all') to 5 ('most of the time'). Sexual desire was treated analogously on a six-point scale ranging from 1 ('no desire') to 6 ('most of the time'). With regard to critical life events, participants were asked to describe these in their own words. Self-constructed items were also used to assess lifestyle factors such as: (1) smoking, which was scored 1 'never smoked', 2 'quitted more than 2 months ago' and 3 'currently smoking'; (2) alcohol drinking (1 'frequently', 2 'seldom or not'); (3) physical exercise (1 'never' to 5 'very often'); and (4) watching television (1 'never' to 5 'very often').

Statistics Mean values are presented as means \pm SD. Sociodemographic variables were evaluated by analyses of $\chi^{2}$ tests (e.g. smoking), $t$ tests, Mann-Whitney $U$ test and ANOVA as appropriate. Multivariate three-factorial ANOVA (MANOVA) were calculated in order to control for type I errors. The independent variables were sex (men, women), treatment (GLA, insulin) and age $(<65$ years, $\geq 65$ years), the dependent variables were the anthropometric and psychological variables. In case of lacking multivariate normality (in terms of type I error rate, there is a general consensus that MANOVA is a robust procedure), significant effects were proven by non-parametric analyses using Mann-Whitney $U$ tests. If no changes in significance were found, MANOVA results of means and SD are reported. The hypothesis that glucometabolic control $\left(\mathrm{HbA}_{1 \mathrm{c}}\right)$ can be predicted was proven by multiple linear regression analyses separately for men and women. One reason for calculating sex-specific multiple regressions was the assumption that sex-related differences exist in anthropometric and psychological aspects; the second reason was the assumption that there are different predictors for men and women in sexual behaviour, and the fact that these were considered to be important. The model contained sociodemographic (age, sex), disease-specific (treatment, diabetes duration, diabetes education, long-term complications), clinical and anthropometric (BMI, FPG, HDL-cholesterol, LDL-cholesterol, triacylglycerol, SBP, DBP), and psychological (compliance, diabetes knowledge, NHP, ICP-D1, FKV, MAS, 
BSSS, DSTQ, sexual desire, satisfaction and for men erectile function) variables as predictors. Statistical analyses were performed with SPSS 10.0 for Windows. $p$ values of less than 0.05 were considered to indicate significant differences.

\section{Results}

Study population Of the 257 type 2 diabetes patients, $47 \%$ (60 men/62 women) were treated with insulin, with $24 \%$ of these patients on an intensive regimen with basal and shortacting insulin. The remaining 53\% (66/69) were being treated with GLA and were younger than insulin-treated patients $(p=0.019)$ (Table 1). Some 43\% (54/56) were younger than 65 years, while $57 \%(72 / 75)$ were at least 65 years old $(p=0.986)$. In the younger group, 51 patients were being treated with insulin and 59 with GLA, whereas in the older group 71 patients were on insulin and 76 on GLA $(p=0.785)$. As expected, the known diabetes duration was longer in insulin-treated and in older patients. Women were less well educated $(p=0.0001)$ and more often homemakers or unemployed than men $(p=0.005)$. They were also more likely to be living alone as singles, divorcees or widows $(p=0.012)$.

Due to the regional structure of diabetes education, patients on insulin had attended more diabetes education courses than those on GLA (2.55 vs $1.59, p<0.01)$. During the year prior to the study, no differences between men and women were reported concerning perception and experience of hyperglycaemia, as well as numbers of hospital admissions or events of metabolic deterioration. Diabetesrelated complications were present in 130 patients $(50 \%)$, of which $70(27 \%)$ were on insulin and $60(23 \%)$ on GLA treatment with no significant difference between the sexes.

Anthropometric and metabolic data Anthropometric and metabolic data were compared by MANOVA in relation to sex (men, women), treatment (GLA, insulin) and age $(<65, \geq 65)$. The multivariate main effects of $\operatorname{sex}\left(F_{16,23}=13.64, p<0.001\right)$, treatment $\left(F_{16,23}=9.15, p<0.001\right)$ and age $\left(F_{16,23}=2.30\right.$, $p=0.004)$, but no interactions showed significant differences. There were only few differences between sex, treatment and age subgroups in anthropometric and metabolic variables (Table 1). As expected, women had higher HDL-cholesterol $(p=0.01)$ and lower DBP $(p=0.02)$, but also higher insulin sensitivity as assessed by QUICKI $(p=0.04)$. In women, $\mathrm{HbA}_{1 \mathrm{c}}$ and $\mathrm{BMI}$ tended to be higher, but did not significantly differ compared with men ( $p=0.07$ and 0.08 , respectively). Women patients exhibited weak positive relationships of $\mathrm{HbA}_{1 \mathrm{c}}$ with BMI $(r=0.646, p<0.001)$ and triacylglycerol $(r=0.20, p=0.001)$. Patients on GLA had shorter known diabetes duration $(p=<0.001)$, lower $\mathrm{HbA}_{1 \mathrm{c}}(p=0.001)$ and FPG $(p=0.011)$ and were more insulin-sensitive $(p=0.039)$. There was a negative relationship between QUICKI and triacylglycerol:HDL-cholesterol ratio $(r=-0.238, p<0.01)$; the triacylglycerol:HDL-cholesterol ratios were not different between the treatment groups. Older patients ( $\geq 65$ years) had longer known diabetes duration $(p<0.001)$, better $\mathrm{HbA}_{1 \mathrm{c}}$ $(p=0.038)$ and lower FPG $(p=0.046)$, LDL-cholesterol $(p=0.023)$, DBP $(p=0.006)$ and triacylglycerol:HDLcholesterol ratio $(p=0.028)$, but higher HDL-cholesterol $(p=0.008)$ (Table 1$)$.

Table 1 Clinical characteristics and anthropometric and fasting metabolic data of 257 outpatients with type 2 diabetes mellitus on insulin or treatment with GLA

\begin{tabular}{|c|c|c|c|c|c|c|c|c|c|}
\hline Variable & Men & Women & $p$ value & Insulin & GLA & $p$ value & Age $<65$ years & Age $\geq 65$ years & $p$ value \\
\hline$n$ & 126 & 131 & & 122 & 135 & & 110 & 147 & \\
\hline Age (years) & $65 \pm 9$ & $65 \pm 10$ & NS & $65 \pm 9$ & $64 \pm 9$ & 0.019 & $56 \pm 5$ & $71 \pm 5$ & 0.000 \\
\hline Diabetes duration (years) & $13 \pm 9$ & $15 \pm 9$ & NS & $17 \pm 9$ & $11 \pm 6$ & 0.000 & $11 \pm 6$ & $16 \pm 10$ & 0.000 \\
\hline Waist circumference $(\mathrm{cm})$ & $111 \pm 9$ & $110 \pm 11$ & NS & $111 \pm 10$ & $110 \pm 10$ & NS & $111 \pm 10$ & $110 \pm 10$ & NS \\
\hline BMI $\left(\mathrm{kg} / \mathrm{m}^{2}\right)$ & $30 \pm 4$ & $31 \pm 5$ & NS & $30 \pm 5$ & $30 \pm 5$ & NS & $30 \pm 5$ & $30 \pm 5$ & NS \\
\hline $\mathrm{HbA}_{1 \mathrm{c}}(\%)$ & $7.4 \pm 1.2$ & $7.7 \pm 1.3$ & NS & $7.8 \pm 1.0$ & $7.3 \pm 1.4$ & 0.001 & $7.7 \pm 1.4$ & $7.4 \pm 1.3$ & 0.038 \\
\hline FPG $(\mathrm{mmol} / \mathrm{l})$ & $8.8 \pm 3.1$ & $8.9 \pm 3.0$ & NS & $9.3 \pm 3.3$ & $8.4 \pm 2.8$ & 0.011 & $9.3 \pm 3.3$ & $8.5 \pm 2.8$ & 0.046 \\
\hline HDL-cholesterol (mmol/l) & $1.4 \pm 0.3$ & $1.6 \pm 0.4$ & 0.011 & $1.5 \pm 0.4$ & $1.5 \pm 0.3$ & NS & $1.5 \pm 0.3$ & $1.6 \pm 0.4$ & 0.008 \\
\hline LDL-cholesterol (mmol/1) & $2.7 \pm 0.9$ & $2.6 \pm 1.0$ & NS & $2.6 \pm 0.9$ & $2.7 \pm 0.8$ & NS & $2.8 \pm 0.9$ & $2.6 \pm 0.7$ & 0.023 \\
\hline Triacylglycerol (mmol/1) & $1.8 \pm 1.0$ & $1.8 \pm 0.9$ & NS & $1.8 \pm 0.9$ & $1.9 \pm 0.9$ & NS & $2.0 \pm 1.0$ & $1.8 \pm 0.8$ & NS \\
\hline $\mathrm{SBP}(\mathrm{mmHg})$ & $135 \pm 15$ & $134 \pm 14$ & NS & $135 \pm 14$ & $134 \pm 14$ & NS & $134 \pm 16$ & $134 \pm 13$ & NS \\
\hline DBP (mmHg) & $79 \pm 9$ & $77 \pm 9$ & 0.022 & $77 \pm 9$ & $79 \pm 9$ & NS & $80 \pm 9$ & $77 \pm 9$ & 0.006 \\
\hline QUICKI & $0.30 \pm 0.04$ & $0.31 \pm 0.05$ & 0.039 & $0.29 \pm 0.05$ & $0.31 \pm 0.03$ & 0.001 & $0.30 \pm 0.05$ & $0.30 \pm 0.03$ & NS \\
\hline Triacylglycerol:HDL-cholesterol & $3.1 \pm 1.8$ & $2.9 \pm 1.6$ & NS & $2.9 \pm 1.6$ & $3.1 \pm 1.7$ & NS & $3.3 \pm 1.8$ & $2.8 \pm 1.5$ & 0.028 \\
\hline
\end{tabular}

Values are means $\pm \mathrm{SD}$ 
Diabetes-related knowledge, psychological and social factors Diabetes knowledge was analysed separately for patients on GLA and insulin. In insulin-treated patients no sex-related differences appeared, whereas women on GLA had better knowledge than men on GLA $(p=0.012)$, particularly regarding nutrition and dietary issues.

There were sex-related differences in lifestyle factors. Thus more women patients had never smoked $(32 \%$ vs $21 \%)$ and fewer women were previous smokers $(9 \%$ vs $20 \%, p<0.001$ each), but no differences were seen with regard to current smoking status ( $8 \%$ vs $10 \%)$. Treatmentrelated differences were not noted with regard to smoking, but age-related differences were noted. Fewer younger patients had never smoked (39\% vs 63\%) and more were still smoking ( $30 \%$ vs $9 \%, p<0.001$ each), whereas the numbers of those who had stopped smoking were comparable $(31 \%$ vs $29 \%$ ). More women than men reported no or very rare alcohol consumption ( $70 \%$ vs $47 \%, p \leq 0.001)$ and more women than performed regular physical exercise $(20 \%$ vs $12 \%, p<0.001)$. However, fewer than half the patients worked-out regularly (38\% men vs $30 \%$ women). Watching television was a frequent leisure activity in all subgroups (mean 3.7 on a scale from 1-never to 5-very often). All these differences occurred independently of treatment regimen and age.

On average, patients reported $1.2 \pm 1.0$ life events as sources of stress in the year before this study. Younger patients recorded more such events than older patients (1.7 vs $0.9, p<0.001)$. Psychological test batteries were analysed by MANOVAs; when effects were proven multivariate significant, univariate means were interpreted. Testing PLD and partnership satisfaction (MAS) did not reveal multivariate differences between sex, treatment and age. Coping with the disease indicated multivariate significance for sex $\left(F_{5,249}=4.00 ; p=0.002\right)$ in that, regardless of the mode of treatment, women patients used more and different strategies to deal with their diabetes, in particular more religiosity, active coping and distraction $(p=0.0001, p=0.048, p=0.007$, respectively). No sex-related differences were found for depressive coping, trivialising and wishful thinking efforts. On the other hand, women with type 2 diabetes mellitus tended to express a lower degree of satisfaction with social support in the BSSS ( $p=0.034$; BSSS multivariate: $F_{4,224}=$ 2.14, $p=0.07$ ). They also tended to rely more on external control provided by their doctor ( $p=0.057$; ICP-D1 multivariate: $\left.F_{4,250}=2.17, p=0.07\right)$ and on unpredictability $(p=0.013)$. Overall, there were no sex-related differences concerning satisfaction with therapy in this type 2 diabetes cohort. Insulin-treated patients presented with higher therapy satisfaction $(p=0.007)$ than patients on GLA. However, compliance with the prescribed therapy was not different across all groups. Sexual desire and sexual satisfaction differed between men and women $(p<0.001)$, as well as between older and younger patients $(p<0.001)$, suggesting more desire in men and younger patients, and more satisfaction in women and older patients. Analysis of $\operatorname{sex} \times$ treatment interaction displayed higher satisfaction in men on GLA and in women on insulin $(p=0.003)$. Older men reported more erection problems than younger men $(p<0.001)$.

Predictors of metabolic control Even in the absence of major sex-related differences in metabolic and sociopsychological variables in this sample, one might hypothesise that different factors contribute to prediction of glucometabolic control in men and women. Given that erectile problems in men could also be an important aspect of metabolic control, separate multiple regression analyses were performed. We entered all predictors together in the analyses, but also proved outcomes by stepwise multiple regression analyses, which revealed the same results. $\mathrm{HbA}_{1 \mathrm{c}}$ could be predicted in both $\left(R^{2}=0.737, p=0.012\right)$ and women $\left(R^{2}=0.597, p=0.019\right)$, explaining about 73 and $60 \%$ of variance respectively, but by a distinct pattern of metabolic and psychological variables (Table 2). Against our hypothesis, no sociodemographic variables such as age and diabetes duration reached statistical significance, while anthropometric and clinic variables only did so in women;.

Table 2 Predictors of $\mathrm{HbA}_{1 \mathrm{c}}$ in male and female outpatients with type 2 diabetes mellitus

\begin{tabular}{|c|c|c|}
\hline Predictors of $\mathrm{HbA}_{1 \mathrm{c}}$ & Beta & $p$ value \\
\hline \multicolumn{3}{|l|}{ Women } \\
\hline Long-term complications & 0.539 & 0.004 \\
\hline Therapy compliance ${ }^{\mathrm{a}}$ & 0.688 & 0.001 \\
\hline BMI & 0.539 & 0.050 \\
\hline Sexual desire ${ }^{b}$ & -0.673 & 0.002 \\
\hline Pain (NHP) & -0.229 & 0.003 \\
\hline Social isolation (NHP) & 0.840 & 0.008 \\
\hline Emotional reactions (NHP) & -0.447 & 0.008 \\
\hline Insomnia (NHP) & 0.484 & 0.027 \\
\hline Internal control (ICP-D1) & -0.380 & 0.039 \\
\hline External control by chance (ICP-D1) & -0.545 & 0.019 \\
\hline External control by doctor (ICP-D1) & 0.387 & 0.053 \\
\hline Depressive coping (FKV) & 0.772 & 0.031 \\
\hline \multicolumn{3}{|l|}{ Men } \\
\hline Erectile function $^{\mathrm{c}}$ & -0.381 & 0.066 \\
\hline Emotional support (BSSS) & 0.516 & 0.007 \\
\hline Satisfaction with support (BSSS) & -0.309 & 0.054 \\
\hline Instrumental support (BSSS) & -0.327 & 0.066 \\
\hline Hyperglycaemia (DTSQ) & 0.465 & 0.018 \\
\hline Hypoglycaemia (DTSQ) & -0.491 & 0.015 \\
\hline
\end{tabular}

\footnotetext{
${ }^{\mathrm{a}}$ Single item to measure compliance

${ }^{\mathrm{b}}$ Two-item scale to measure sexual desire

${ }^{\mathrm{c}}$ Two-item scale to measure erectile function
} 
In women mainly quality of life (NHP) and locus of control (ICP-D1) were associated with $\mathrm{HbA}_{1 \mathrm{c}}$. Long-term complications, BMI and compliance were also positively related to $\mathrm{HbA}_{1 \mathrm{c}}$. Lower sexual desire, lower quality of life (NHP: less pain and emotional reactions and more social isolation and insomnia) and depressive coping strategies, as well as less pronounced internal control beliefs and belief in chance were significantly associated with poorer metabolic control (Table 2).

In men patients, social support (BSSS) and satisfaction with diabetes treatment (DTSQ) were prominent in predicting metabolic control. High emotional support and both DTSQ items covering experience of hyperglycaemia and hypoglycaemia predicted metabolic control. As expected, fewer hypo- and more hyperglycaemic episodes were associated with higher $\mathrm{HbA}_{1 \mathrm{c}}$. Some aspects nearly reached significance. As in women, sexuality was related to $\mathrm{HbA}_{1 \mathrm{c}}$ and a higher degree of erectile dysfunction was associated with worse metabolic control. Two support scales of the BSSS, namely satisfaction with support and instrumental support, were also negatively related to $\mathrm{HbA}_{1 \mathrm{c}}$ (Table 2).

\section{Discussion}

This outpatient population was homogenous with regard to anthropometric variables describing an overweight to obese, slightly hypertriacylglycerolaemic and hypertensive cohort. Mean $\mathrm{HbA}_{1 \mathrm{c}}$ levels of $7.4 \%$ in men and $7.7 \%$ in women showed that current targets of glycaemic control [20] were not completely met, which probably results from the local patient referral system. The hospital's outpatient service serves as a tertiary care centre dealing mostly with patients who are either considered difficult to treat by their primary or secondary care providers or are only treated for short time periods until targets are reached. Metabolic control was only slightly different between sexes, but tended to be worse in women. Thus, we did not confirm previous studies showing that men have lower $\mathrm{HbA}_{1 \mathrm{c}}$ levels and/or fewer episodes of ketonuria [21]. Such results were claimed to be a consequence of more intensive care for men [22].

$\mathrm{HbA}_{1 \mathrm{c}}$ was related to the degree of insulin resistance and indicators of insulin resistance such as abdominal obesity (waist circumference) and circulating lipids (triacylglycerol, HDL-cholesterol). We confirmed the robust negative correlation between measures of insulin sensitivity (QUICKI, triacylglycerol:HDL-cholesterol ratio) [11]. Interestingly, insulin sensitivity was not different between sexes, although differences in sex hormones, adipocytokines [23, 24] and/or abdominal obesity [25] could lead to sex-specific alterations in insulin signalling and action.

Only a few previous studies have addressed sex-specific aspects of quality of care [26]. Here we report slightly lower DBP and higher HDL-cholesterol in women with type 2 diabetes, whereas other cardiovascular risk factors were identical in both sexes. Previous studies have found worse BP control, higher SBP [22] and higher LDLcholesterol in women patients [26]. It has therefore been suggested that women might be receiving lower-quality treatment [27], possibly contributing to their excess cardiovascular morbidity and mortality rates [3, 28]. However, we detected only minor, clinically irrelevant changes in cardiovascular risk factors and no differences in diabetes-related complications between men and women.

This study further analysed clinical and psychological variables that have been suggested to affect $\mathrm{HbA}_{1 \mathrm{c}}$ and quality of life [7]. Despite similar metabolic control, women patients on GLA proved to have more knowledge of diabetes than men treated with GLA. Likewise, insulin-treated men and women patients had higher $\mathrm{HbA}_{1 \mathrm{c}}$ values, although they had attended more diabetes education training sessions than patients on GLA. This clearly indicates that disease-specific knowledge is neither the only, nor even the major factor defining glucometabolic control. Other [29], but not all studies [12, 30] have also failed to find a relationship between diabetes knowledge and metabolic control. Of note, these studies do not justify negating the use of basic knowledge about diabetes care or the necessity of diabetes education, which are also intended to improve the patient's motivation to comply with treatment regimens.

Women patients used more and different strategies for coping with their disease, but claimed less satisfaction with social support. In addition, the women in the present study more frequently lived alone and had lower socioeconomic status than the men. Because the sex distribution was nearly identical between the age groups, this finding cannot be explained by an age effect. Positive social support, particularly by the spouse, was found to improve glycaemic control and better adaptation to the disease [31]. In our study, however, too much emotional support for men seemed to be associated with worse glycaemic control. Our women patients also tended to have more negative perceptions and emotions, which is in line with studies reporting a two- to threefold increased prevalence of depression in women compared with men patients [7, 26]. Depression not only increases the burden of disease and independently impairs quality of life, but also contributes to increased mortality rates in type 2 diabetes [32]. Taken together, it is tempting to speculate that the lower degree of social support, quality of life and socioeconomic status, as well as the increased prevalence of symptoms of anxiety and depression might have prevented the women patients from achieving improved glucose control despite having better diabetes-specific knowledge and coping efforts than men.

The prediction of insufficient glucometabolic control as assessed by $\mathrm{HbA}_{1 \mathrm{c}}$ reflected many aspects of the findings 
discussed above. Social isolation, reduced sexual desire, insomnia and depressive coping strategies were strong predictors of high $\mathrm{HbA}_{1 \mathrm{c}}$ in women. Interestingly, high selfrated therapy adherence and dependence on doctor's control also related to poorer metabolic control in women. This is partly in contrast to suggestions of lower compliance at least in insulin-treated women patients [33]. We did not note any sex-, treatment- or age-related differences in compliance and satisfaction with diabetes therapy and management.

Perception and experience of hyperglycaemia, as well as the number of hospital admissions or episodes of metabolic deterioration were not different between sexes, a finding which is similar to reports on hospital admissions for uncontrolled diabetes [34]. Another study found higher frequencies of diabetes-related admissions in men than in women, which they explained by variances in care-seeking behaviour [35]. In our men patients, the number of hyperglycaemic and hypoglycaemic experiences was among the best predictors of glucometabolic control. This finding indicates that self-perception and blood glucose reporting seemed to be adequate in our male cohort. Aside from emotional support, other factors were only weak predictors of $\mathrm{HbA}_{1 \mathrm{c}}$ in men. Erectile dysfunction also tended to relate to metabolic control. Men displayed stronger sexual desire and activity, and less satisfaction with their sex life than women, which has been discussed elsewhere in detail [36]. Interestingly, impaired glucose control may initiate a vicious circle leading to erectile dysfunction, which in turn could cause negative emotions, thereby further impairing glucose control.

There are some limitations to this study. Thus the data cannot be extrapolated to patients treated in primary or secondary care settings, because the hospital's outpatient service serves as a tertiary care centre. Thus, the population consists of referral patients, mostly patients considered difficult to treat by their primary or secondary care providers, or patients only treated for a short time period within the centre until treatment targets are achieved. Consequently, the results cannot be extrapolated to general populations. Furthermore, the conclusions are restricted to patients with reasonable attendance records and the ability to fill in questionnaires. Individuals less compliant with treatment and lifestyle modifications are also less likely to be included in such studies. The overall number of participants could be considered rather low, but this is counterbalanced by the homogeneity of the study population with regard to social background and by the extensive socio-psychological testing.

\section{Conclusion}

Patients with type 2 diabetes mellitus from a metropolitan diabetes outpatient service exhibited only minor sex- specific differences in control of metabolic variables and cardiovascular risk factors. However, individual coping with the disease was clearly different between both sexes. Women reported more negative emotions and impaired quality of life, but showed more efforts to deal with their disease. They trusted in their doctor's support, while at the same time being more likely to experience their disease as unforeseeable. Thus, women patients could benefit both from support of their willingness to influence their diabetes and from more individualised diabetes care. On the other hand, men are more satisfied with their social support, but could benefit from intensified education courses focusing on diabetes-related knowledge. Based on these results and conclusions, sex-specific strategies need to be tested in order to improve diabetes management.

Acknowledgements This study was supported partly by the European Foundation for the Study of Diabetes (EFSD grants through Novo Nordisk and GlaxoSmithKline to M. Roden).

Duality of interest The authors declare that there is no duality of interest associated with this manuscript.

\section{References}

1. Zimmet P, Alberti KG, Shaw J (2001) Global and societal implications of the diabetes epidemic. Nature 414:782-787

2. Schroeder SA (2007) Shattuck Lecture. We can do betterimproving the health of the American people. N Engl J Med 357:1221-1228

3. Huxley R, Barzi F, Woodward M (2006) Excess risk of fatal coronary heart disease associated with diabetes in men and women: meta-analysis of 37 prospective cohort studies. BMJ 332:73-78

4. Thorand B, Baumert J, Kolb H et al (2007) Sex differences in the prediction of type 2 diabetes by inflammatory markers: results from the MONICA/KORA Augsburg case-cohort study, 19842002. Diabetes Care 30:854-860

5. Gouni-Berthold I, Berthold HK, Mantzoros CS, Bohm M, Krone W (2008) Gender disparities in the treatment and control of cardiovascular risk factors in type 2 diabetes. Diabetes Care 31:1389-1391

6. Hermanns N, Kulzer B, Krichbaum M, Kubiak T, Haak T (2005) Affective and anxiety disorders in a German sample of diabetic patients: prevalence, comorbidity and risk factors. Diabet Med 22:293-300

7. Goldney RD, Phillips PJ, Fisher LJ, Wilson DH (2004) Diabetes, depression, and quality of life: a population study. Diabetes Care 27:1066-1070

8. Glasgow RE, Anderson RM (1999) In diabetes care, moving from compliance to adherence is not enough. Something entirely different is needed. Diabetes Care 22:2090-2092

9. Norris SL, Engelgau MM, Narayan KM (2001) Effectiveness of self-management training in type 2 diabetes: a systematic review of randomized controlled trials. Diabetes Care 24:561-587

10. Luszczynska A, Boehmer S, Knoll N, Schulz U, Schwarzer R (2007) Emotional support for men and women with cancer: do patients receive what their partners provide. Int $J$ Behav Med 14:156-163

11. Brehm A, Pfeiler G, Pacini G, Vierhapper H, Roden M (2004) Relationship between serum lipoprotein ratios and insulin resistance in obesity. Clin Chem 50:2316-2322 
12. Hermanns N (1996) Entwicklung und Evaluation eines Wissenstests für nicht-insulinpflichtige Typ-IIb-Diabetiker. Diabetes und Stoffwechsel 5:183-190

13. Roth R, Teupe B, Borkenstein M (1996) Der DiabetesWissenstest: Typ-I (DWT: Typ-I). Hogrefe, Göttingen

14. Bengel J, Beutel M, Broda M et al (2003) Chronic diseases, psychological distress and coping - challenges for psychosocial care in medicine. Psychother Psychosom Med Psychol 53:83-93

15. Kulzer B, Bauer U, Hermanns H, Bergis KH (1995) Entwicklung eines Problemfragebogens für Diabetiker zur Identifikation von Schulungseinheiten im Umgang mit der Krankheit. Verhaltenstherapie 5(Suppl 1):72

16. Kohlmann CW, Schuler KE, Tausch M (1994) Der IPCFragebogen (IPC-D1). Hans Huber, Bern

17. Hunt SM, McKenna SP (1997) Measuring health status: a new tool for clinicians and epidemiologists. J Royal Coll Gen Pract $35: 185-188$

18. Locke HJ, Wallace KM (1959) Locke-Wallace marital adjustment scale and prediction test: their reliability and validity. Marriage \& Family Living 21:251-255

19. Bradley C (1994) Diabetes Treatment Satisfaction Questionnaire, DTSQ. In: Bradley C (ed) Handbook of psychology and diabetes. Harwood, Chur, pp 111-132

20. Ryden L, Standl E, Bartnik M et al (2007) Guidelines on diabetes, pre-diabetes, and cardiovascular diseases: executive summary. The Task Force on Diabetes and Cardiovascular Diseases of the European Society of Cardiology (ESC) and of the European Association for the Study of Diabetes (EASD). Eur Heart J 28:88-136

21. Nilsson PM, Theobald H, Journath G, Fritz T (2004) Gender differences in risk factor control and treatment profile in diabetes: a study in 229 Swedish primary health care centres. Scand J Prim Health Care 22:27-31

22. Duggirala MK, Cuddihy RM, Cuddihy MT, Nyman MA, Naessens JM, Pankratz VS (2005) Women with diabetes have poorer control of blood pressure than men. J Womens Health (Larchmt) 14:418-423

23. Ding EL, Song Y, Malik VS, Liu S (2006) Sex differences of endogenous sex hormones and risk of type 2 diabetes: a systematic review and meta-analysis. Jama 295:1288-1299
24. Roden M (2006) Mechanisms of disease: hepatic steatosis in type 2 diabetes - pathogenesis and clinical relevance. Nat Clin Pract Endocrinol Metab 2:335-348

25. Banerji MA, Lebowitz J, Chaiken RL, Gordon D, Kral JG, Lebovitz HE (1997) Relationship of visceral adipose tissue and glucose disposal is independent of sex in black NIDDM subjects. Am J Physiol 273:E425-E432

26. Gucciardi E, Wang SC, DeMelo M, Amaral L, Stewart DE (2008) Characteristics of men and women with diabetes: observations during patients' initial visit to a diabetes education centre. Can Fam Physician 54:219-227

27. Legato MJ, Gelzer A, Goland R et al (2006) Gender-specific care of the patient with diabetes: review and recommendations. Gend Med 3:131-158

28. Regitz-Zagrosek V, Lehmkuhl E, Weickert MO (2006) Gender differences in the metabolic syndrome and their role for cardiovascular disease. Clin Res Cardiol 95:136-147

29. Speight J, Bradley C (2001) The ADKnowl: identifying knowledge deficits in diabetes care. Diabet Med 18:626-633

30. Panja S, Starr B, Colleran KM (2005) Patient knowledge improves glycemic control: is it time to go back to the classroom. J Investig Med 53:264-266

31. Trief PM, Grant W, Elbert K, Weinstock RS (1998) Family environment, glycemic control, and the psychosocial adaptation of adults with diabetes. Diabetes Care 21:241-245

32. Zhang X, Norris SL, Gregg EW, Cheng YJ, Beckles G, Kahn HS (2005) Depressive symptoms and mortality among persons with and without diabetes. Am J Epidemiol 161:652-660

33. Rubin R, Peyrot M (1999) Quality of life and diabetes. Diabetes Metab Res Rev 15:205-218

34. Correa-De-Araujo R (2006) Serious gaps: how the lack of sex/ gender-based research impairs health. J Womens Health (Larchmt) 15:1116-1122

35. Washington EL, Shen JJ (2006) Gender discrepancies in the level of disease progression in hospitalized patients with diabetes. Manag Care Interface 19:21-25

36. De Berardis G, Franciosi M, Belfiglio M et al (2002) Erectile dysfunction and quality of life in type 2 diabetic patients: a serious problem too often overlooked. Diabetes Care 25:284-291 